\title{
Comparing the ability of cognitive and affective Theory of Mind in adolescent onset schizophrenia
}

\author{
This article was published in the following Dove Press journal: \\ Neuropsychiatric Disease and Treatment \\ 27 March 2017 \\ Number of times this article has been viewed
}

\author{
Dandan $\mathrm{Li}^{1,2, *}$ \\ Xiaosi $\mathrm{Li}^{3, *}$ \\ Fengqiong $\mathrm{Yu}^{1,2}$ \\ Xingui Chen ${ }^{2,4}$ \\ Long Zhang ${ }^{2,4}$ \\ Dan $\mathrm{Li}^{2,4}$ \\ Qiang Wei ${ }^{2,4}$ \\ Qing Zhang ${ }^{1,2}$ \\ Chunyan Zhu', ${ }^{1,2}$ \\ Kai Wang ${ }^{1,2,4}$
}

'Department of Medical Psychology, Anhui Medical University, Hefei,

${ }^{2}$ Collaborative Innovation Centre

of Neuropsychiatric Disorders and Mental Health, Anhui Province,

${ }^{3}$ Mental Health Center of Anhui

Province, ${ }^{4}$ Department of Neurology,

The First Affiliated Hospital of Anhui

Medical University, Hefei, China

*These authors contributed equally to this work
Correspondence: Chunyan Zhu Medical Psychology Department, Anhui Medical University, Meishan Road, Hefei, 230038, Anhui Province, China

Tel +86 I38 65998867

Fax +86 55I 62923704

Email ayswallow@।26.com
Background: Evidence in the literature suggests that there is an impairment of social cognition in schizophrenia. Theory of Mind (ToM) is defined as one's ability to understand others' wishes, beliefs, intentions, and other psychological states and thereby to judge others' behavior, as an essential component of social cognition. However, there have been limited studies on social cognition, especially ToM in adolescent onset schizophrenia (AOS). The current study aims to investigate ToM abilities in adolescent schizophrenia according to various ToM subcomponents (cognitive ToM and affective ToM) and various ToM orders (first order and second order).

Methods: This study examines ToM in 35 adolescent schizophrenic patients and 35 healthy adolescents using the "Yoni task" and "Faux Pas Recognition test" to assess their affective and cognitive ToM abilities.

Results: In the Yoni task, patients with AOS showed differences in ToM abilities either on a different order or under different conditions. The Faux Pas Recognition task results revealed that AOS patients were not always able to recognize a faux pas or understand complicated emotions under the faux pas scenario. Furthermore, as indicated by the correlation analysis, neither cognitive ToM nor affective ToM was related to the patients' symptoms, disease duration, dose of medication, or intelligence quotient (IQ).

Conclusion: Our findings showed AOS impairment in the performance of ToM tasks. It seemed that impairment in second-order-ToM is more serious. Moreover, these deficits are largely independent of symptom clusters, disease duration, dose of medication, and IQ. It can be speculated that ToM dysfunction may be a hallmark of adolescent schizophrenia.

Keywords: cognitive/affective Theory of mind, Yoni task, Faux Pas Recognition task

\section{Introduction}

During Kraepelin's time, people began to realize that schizophrenia could be found in children. ${ }^{1}$ Schizophrenia can be divided into the following categories by the age of onset: adult schizophrenia (AS: onset aged $>18$ years), adolescent onset schizophrenia (AOS: onset aged 13-18 years), and childhood onset schizophrenia (onset aged $<13$ years). ${ }^{2-4}$ Compared with $\mathrm{AS}$, the following characteristics are observed in AOS: more neurodevelopmental impairments, ${ }^{5}$ more negative symptoms, ${ }^{6}$ and greater frequency of cerebral abnormalities and a longer treatment requirement. ${ }^{7,8}$ The development of AOS often diminishes social networks and results in difficulties in social functioning. ${ }^{9}$ Although impaired social behavior in schizophrenia has been repeatedly described, ${ }^{9}$ the scientific literature on the social cognition of AOS and childhood onset schizophrenia is limited.

Theory of Mind (ToM) damage in AOS may be related to poor social functioning. ${ }^{7,8}$ ToM is defined as an individual's ability to understand others' wishes, beliefs, 
intentions, and other psychological states and to judge others' behavior accordingly, which is an essential component of social cognition. ${ }^{9}$ ToM was divided into cognitive ToM (Cog ToM) and affective ToM (Aff ToM) in the different cognitive processes, ${ }^{10}$ and another important classification is first and second order in the ToM complexity. Cog ToM is used to infer one's own and others' beliefs, intentions, and desires; whereas, affective ToM is used to infer one's own and others' emotional states and feelings. Shamay-Tsoory et al proposed a model in which Cog ToM was a precondition for Aff ToM and Aff ToM processes required both Cog ToM and empathy. ${ }^{11}$ Whereas first-order-ToM is defined as the ability of an individual to understand another person's beliefs, the ability to realize that someone else thinks that a third person believes something is called the "second order" ToM. ${ }^{12}$ Several tasks have been developed to assess the above ToM subcomponents, such as the false belief tasks, ${ }^{13}$ the Yoni task, ${ }^{11}$ the Faux Pas Recognition (FPR) test, ${ }^{14}$ the eye recognition task, and the perspective-taking task. ${ }^{15}$

Adolescence is characterized as a critical period in the development of ToM. ${ }^{16}$ However, initial behavioral evidence showed that Cog ToM and Aff ToM had different developmental trajectories. ${ }^{17,18} \mathrm{Cog}$ ToM always develops earlier than Aff ToM. ${ }^{18}$ Previous research showed that children could pass some simple $\mathrm{Cog}$ ToM tasks (eg, false belief) from the age of 4 to 6 years and gained an understanding of the more complex cognitive tasks at a later age (eg, second belief) between the age of 7 and 9 years. ${ }^{13}$ Developmental findings on Aff ToM processing observed a later developmental trajectory in which Aff ToM began to develop between 7 and 9 years of age and that children between 9 and 11 years could infer complex affective expression (eg, FPR task). ${ }^{17}$

Whether ToM deficits represent a distinctive trait marker or state variables for schizophrenia is debatable. ${ }^{19}$ Several studies found that ToM was related to symptoms, especially in patients in remission who performed significantly better on ToM tests than those in symptomatic periods, ${ }^{20}$ but impairment across ToM domains has been well documented in chronic and first-episode patients, suggesting that ToM impairment is a trait marker in schizophrenia patients. ${ }^{21}$ Additionally, one study reported that schizophrenia showed ToM damage in remission, ${ }^{22}$ first-degree relatives of patients, and high-risk patients and thus might qualify as a stable endophenotype of schizophrenia. ${ }^{21}$ The current study explores whether ToM impairment is a trait phenomenon in the perspective of AOS.

The present study examines the performance of two ToM components (Cog ToM and Aff ToM) in AOS using the
Yoni task and FPR task. The Yoni task is a classic measure to verify the cognitive vs affective aspects of the ToM and to classify first vs second order; its simplicity makes it accessible to children and adolescents. The FPR task is a widely used ToM task to analyze higher-level ToM ability and to investigate Aff and Cog ToM. No studies, to date, have utilized these two tasks in AOS; therefore, we use these two paradigms to explore the defining characteristics of Aff and Cog ToM in AOS.

Considering the effects of the mental disease and the specificity of the age of onset in AOS, the current study aims to explore the following questions: 1) whether ToM deficits exist in late AOS compared with healthy adolescent controls and 2) whether the deficits are related to symptoms and disease duration. We hypothesized that patients with late AOS would perform less well in ToM performance, compared with health controls. We also hypothesized that there was no association between ToM performance and the illness course and symptoms.

\section{Methods}

\section{Subjects}

Thirty-five adolescents with schizophrenia and 35 demographically similar healthy volunteers participated in this study. All of the subjects were Chinese patients aged 13-18 years and were consecutively recruited between February 2013 and March 2015 at the Mental Health Center of Anhui Province. The patients were recruited from outpatient clinics for confirmatory diagnostic evaluations by two psychiatrists, and they met the 10th edition of the International Classification of Diseases (ICD-10) diagnostic criteria for schizophrenia. All of the patients were stable in their symptoms and had received treatment for at least 4 months. All patients were stable on fixed doses of medication (Table 1). In most cases, the psychological and psychiatric assessments were evaluated in the same day. The psychological assessments were completed by a graduate student in clinical psychology after training, and psychiatric assessments were assessed by experienced psychiatrists. Healthy adolescents were recruited from local junior and senior high schools and were age-, gender-, and intelligence-matched. The exclusion criteria for all subjects were a history of drug and alcohol abuse, head trauma, seizure disorder, other major neurological disorder, mental retardation or a score on the 17-item Hamilton Depression Rating Scale (HDRS) of $>7 .{ }^{23}$ All participants were right-handed.

All of the subjects and their parents provided assent/ consent to participate. The study was approved by the Ethics Committee of Anhui Medical University. 
Table I Differences between the groups in terms of demographics, clinical, and neuropsychological performance and medication

\begin{tabular}{|c|c|c|c|c|c|c|}
\hline \multirow[t]{2}{*}{ Variables } & \multicolumn{2}{|l|}{$\operatorname{AOS}(n=35)$} & \multicolumn{2}{|l|}{ HC $(n=35)$} & \multirow[t]{2}{*}{$t$} & \multirow[t]{2}{*}{$P$-value } \\
\hline & Mean (SD) & Range & Mean (SD) & Range & & \\
\hline \multicolumn{7}{|l|}{ Demographic } \\
\hline Age (years) & $16.48(1.42)$ & $13-18$ & $16.31(1.21)$ & $14-18$ & 0.54 & 0.59 \\
\hline Gender (M/F) & $20 / 15$ & & $20 / 15$ & & & \\
\hline Education (years) & $9.68(1.18)$ & $8-12$ & $10.17(1.27)$ & $9-12$ & -1.65 & 0.10 \\
\hline \multicolumn{7}{|l|}{ Clinical } \\
\hline Duration of illness (years) & $1.32(1.23)$ & $0-6$ & & & & \\
\hline HARS & $4.91(3.36)$ & $0-18$ & $4.65(4.26)$ & $0-18$ & 0.28 & 0.78 \\
\hline HDRS & $3.54(2.92)$ & $0-13$ & $2.97(3.14)$ & $0-13$ & 0.78 & 0.43 \\
\hline CPZ equivalents (mg) & $439.14(117.65)$ & $150-800$ & & & & \\
\hline \multicolumn{7}{|l|}{ PANSS subscales } \\
\hline Positive symptoms & $13.17(6.88)$ & $7-27$ & & & & \\
\hline Negative symptoms & $12.08(6.74)$ & $7-40$ & & & & \\
\hline General symptoms & $22.78(8.85)$ & $16-39$ & & & & \\
\hline Total & $48.02(15.75)$ & $31-90$ & & & & \\
\hline \multicolumn{7}{|l|}{ Neuropsychological tasks } \\
\hline IQ-Raven (correct number) & $50.11(5.90)$ & $40-59$ & $50.57(4.55)$ & $40-59$ & -0.36 & 0.72 \\
\hline SCWT-A (s) & $16.93(3.89)$ & $10.45-25.45$ & $|3.2|(2.4 \mid)$ & $10.47-19.16$ & 4.46 & $<0.001 * * *$ \\
\hline SCWT-B (s) & $21.28(7.44)$ & $10.58-46.88$ & I5.73 (3.74) & $10.58-24.30$ & 3.95 & $<0.001 * * *$ \\
\hline SCWT-C (s) & 31.61 (1I.87) & $10-77.58$ & $24.76(8.03)$ & $10.00-51.00$ & 2.79 & $0.01 * *$ \\
\hline Verbal Fluency Test & $65.41(10.92)$ & $32-75$ & $65.03(17.19)$ & $45-98$ & 0.07 & 0.94 \\
\hline Digit span (forward) & $7.91(0.29)$ & $6-8$ & $7.88(0.32)$ & $7-8$ & -1.44 & 0.16 \\
\hline Digit span (backward) & $5.18(1.46)$ & $3-6$ & $5.76(1.24)$ & $4-7$ & $-|.5|$ & 0.14 \\
\hline \multicolumn{7}{|l|}{ Yoni task } \\
\hline First-order-physical-condition & $6.82(1.97)$ & $\mathrm{I}-8$ & $7.14(1.92)$ & $\mathrm{I}-8$ & 0.45 & 0.50 \\
\hline Second-order-physical-condition & $8.62(1.61)$ & $4-11$ & $9.08(1.46)$ & $5-11$ & 1.55 & 0.22 \\
\hline \multicolumn{7}{|l|}{ Faux Pas Recognition Test } \\
\hline Control questions (physical condition) & $9.82(0.45)$ & $8-10$ & $9.97(0.17)$ & $9-10$ & 3.06 & 0.09 \\
\hline
\end{tabular}

Notes: $* * p<0.01 ; * * * p<0.001$.

Abbreviations: CPZ, chlorpromazine; HARS, Hamilton Anxiety Rating Scale; HDRS, Hamilton Depression Rating Scale; IQ, intelligent quotient; PANSS, Positive and Negative Syndrome Scale; PANSS sum, scores of the positive and negative syndrome scale; PANSS positive, the score of the positive syndrome scale in PANSS; PANSS negative, the score of the negative syndrome scale in PANSS; SCWT-A, B, or C (s), the mean of response time of the part A, B, or C in the Stroop-Color Word Test; SD, standard deviation; AOS, adolescent onset schizophrenia; HC, healthy controls.

\section{Measurements}

\section{Clinical and neuropsychological background assessments}

AOS was evaluated with the 14-item Hamilton Anxiety Rating Scale (HARS), ${ }^{24}$ the 17 -item HDRS, and the Positive and Negative Syndrome Scale (PANSS) by a trained psychiatrist. ${ }^{23,25}$ The PANSS was used to evaluate anxiety, depression, and the presence/absence or severity of the positive, negative, and general psychopathology of schizophrenia. The HARS and HDRS were used to assess the anxiety and depression of the patients. All patients received one type of atypical antipsychotics (Aripiprazole, Clozapine, Risperidone, Quetiapine), and the medication dosages have been transformed into chlorpromazine equivalents. ${ }^{26}$ Additionally, the subjects' intelligence quotients (IQs) were assessed using the Standard Progressive Matrices of Raven. The interference task of the Stroop Color-Word Test (SCWT) was used to assess the subjects' response inhibition and interference susceptibility. The score included three parts as follows: part A (word condition), part B (color condition), and part $\mathrm{C}$ (word-color interference condition). Part $\mathrm{C}$ mainly assesses executive function. In the Verbal Fluency Test (VFT) they had to separately name six classes of words (animals, vegetables, appliances, fruit, words beginning with water, words beginning with self), as many as possible, within $1 \mathrm{~min}$, then the total number of the subject responses was calculated. ${ }^{27}$ VFT assessed frontal lobe function, Digit Span Test assessed working memory and short-term memory, which has forward and backward parts. The total score was recorded.

\section{Yoni task}

The Chinese version of this task (programmed using E-prime) consisted of 96 trials based on a task as previously described. Each trial had a cartoon outline of a face (named Xiao Ming) and four colored pictures, each of which was 
located in one corner of the computer screen. The pictures were of objects (eg, fruit or chairs) or faces. Based on the cues available (such as Xiao Ming's eye gaze and facial expression) at the top of the screen, the subject's task was to point to the correct answer (according to the cartoon to which Xiao Ming was referring). After a participant found the correct answer, he or she was asked to respond (click) quickly. These trials fall into the following three classes: cognitive, affective and physical. The "affective" trials investigate "hot" ToM, the "cognitive" trials inspect "cold" ToM, and the physical trials act as the control condition to explore whether the subject understood the questions. All of the trials were further subdivided into first and second order inferences. The addition would have no influence on the difficulty and framework of the task, which were basically the same as the original. The duration of the task was approximately 12 minutes, and E-prime recorded the participant's correct numbers and reaction times. The individual received one point for each correct answer. The task consisted of three parts:

- Cognitive condition

The first-order-cognitive-condition, 12 trials (on a scale from 0 to 12; translated from Chinese)

Cog 1: Xiao Ming is thinking of $?$

The second order cognitive condition, 12 trials (on a scale from 0 to 12 ; translated from Chinese)

Cog2: Xiao Ming is thinking of the toy that wants?

- Affective condition

The first-order-affective-condition, 12 trials (on a scale from 0 to 12; translated from Chinese)

Aff1: Xiao Ming likes

The second order affective condition, 42 trials (on a scale from 0 to 42; translated from Chinese)

Aff2: Xiao Ming likes animals that likes?

- Physical condition

The first-order-physical-condition, 8 trials (on a scale from 0 to 8 ; translated from Chinese)

Phy1: Xiao Ming is near to ?

The second-order-physical-condition, 12 trials (on a scale from 0 to 12 ; translated from Chinese)

Phy2: Xiao Ming has the same fruit that has?

In our view, the second order Aff ToM is a complex item and thus the 16 questions may not fully reflect subjects' abilities in the second order Aff ToM. Therefore, we added 30 questions to the second order Aff ToM condition (eg, original question: Xiao Ming likes animals that likes?; added question: Xiao Ming likes toys that likes?).

\section{FPR test}

The Baron-Cohen's FPR test was applied in the Chinese context. The Chinese version of the FPR test has good testretest reliability and interrater reliability. ${ }^{28} \mathrm{~A}$ faux pas is considered to occur if a person says something unintentionally that should not have been said and the remark makes people feel sad and embarrassed. The following is an example of a social faux pas story in this task:

Liu yan's mother was having a surprise party for Liu yan's birthday. She invited Li mei and said, "Don't tell anyone, especially Liu yan". The day before the party, Li mei and Liu yan were playing together and Li mei ripped her new dress. "Oh!" said Li mei, "I was going to wear this to your party." "What party?" said Liu yan. "Come on", said Li mei, "let's go and see if my mum can mend the tear."

If a subject discovered that there was a faux pas, he or she would realize that the speaker was going to make the listener uncomfortable and predict that those who heard the words would be sad. There were 10 faux pas stories in our study. After each story, five questions were answered:

- Question 1 (Cog ToM: detecting the faux pas): Did someone say something he (or she) should not have said?

- Question 2 (Aff ToM: detecting the faux pas): Who said something he (or she) should not have said?

- Question 3 (Aff ToM: understanding the listener's mental state): Why should he (or she) not have said it?

- Question 4 (Aff ToM: understanding the speaker's mental state): Why did he (or she) say it?

- Question 5 (control condition): Who was the surprise party for?

For scoring, each correct response was given 1 point. If the subject answered "No" to the first question, questions 2 to 4 would be skipped and he (or she) would directly answer question 5. In this a case, questions 1 to 4 were given zero points. The total of "detecting faux pas" (Cog ToM scores) was the sum of the scores for each question 1 of the 10 stories on a scale from 0 to 10 . The total of "understanding (Aff ToM scores)" was the sum of the scores for questions 2 to 4 of the 10 stories on a scale from 0 to 30 . The total score for questions 1 to 4 was regarded as the faux pas total. Question 5 was a control condition to examine whether the subjects understood the story and was scored on a scale from 0 to 10 .

\section{Statistical analysis}

Statistical Package for the Social Sciences (SPSS) 19.0 was used for the statistical analysis. Independent sample $t$-tests were used to examine the differences in independent variables 
(age, education, duration of illness, subcomponent scores, and clinical and neuropsychological background assessments). To assess performance in the ToM tasks, a repeatedmeasures ANOVA was respectively performed on the first and second-order-ToM, ToM components (three components: cognitive, affective and control subcomponents) acted as within-subject factors and the groups (AOS and healthy controls [HC] group) acted as between-subject factors. The data were used to detect the significance of the main effects (groups and ToM components) and the interaction effect between the groups and ToM components. Then, multivariate analysis of variance (MANOVA) was performed on the group difference (AOS and HC group) for each component (Cog ToM, Aff ToM, and physical condition). MANOVA was conducted on Faux Pas Recognition Test, which was used to examine the group differences in the components. We also used Pearson's correlation analysis to examine the correlation between the severity of symptoms, duration of illness, SCWT performance, and ToM subcomponents. The threshold of statistical significance was set at $P<0.05$.

\section{Results}

\section{Demographic and clinical characteristics and neuropsychological results}

The background and neuropsychological data of AOS vs the healthy controls are shown in Table 1 . The results of the independent-sample $t$-test indicated that there was no significant difference in terms of age $(P=0.59)$, years of education $(P=0.10)$, HARS $(P=0.78)$ and HDRS scores $(P=0.43)$, and IQ-Raven $(P=0.72)$ between the groups. A significant difference was observed between the two groups in the SCWT test (SCWT test A: $P<0.001$; SCWT test B: $P<0.001$; and SCWT test $\mathrm{C}: P=0.01)$. The two groups showed no significant difference in the scores of the VFT $(P=0.94)$, digit span forward $(P=0.16)$ and Digital span backward $(P=0.14)$ tests (Table 1).

\section{Yoni task}

\section{First-order-ToM}

A repeated measures ANOVA was performed on the first-order-ToM using each of the ToM subcomponents (three subcomponents: cognitive, affective, and physical) as the within-subjects factor and the group (EOS group and HC group) as the between-subjects factor. The results demonstrated a primary effect for the ToM condition $\left(F[1.68]=70.103, P<0.001, \eta^{2}=0.508\right)$, and the group-by-condition interaction $(F[1,68]=6.408, P=0.014$, $\left.\eta^{2}=0.086\right)$ reached statistical significance. A multivariate analysis with group as a fixed factor and first physical condition as a covariate was performed to examine the relationship between first cognitive condition and first affective condition. The EOS group had significantly lower scores in first order $\operatorname{Cog} \operatorname{ToM}\left(F[1,68]=4.031, P=0.049, \eta^{2}=0.057\right)$ and first order $\operatorname{Aff} \operatorname{ToM}\left(F[1,68]=6.809, P=0.011, \eta^{2}=0.092\right)($ Figure 1$)$.

\section{Second-order-ToM}

The repeated measures ANOVA on the second-order-ToM was performed for the within-subjects and between-subjects factors of the second-order-ToM as described earlier. There were significant main effects for both the ToM conditions $\left(F[1,68]=5.222, P=0.025, \eta^{2}=0.071\right)$ and the group $\left(F[1,68]=14.772, P<0.001, \eta^{2}=0.178\right)$. There was also a significant effect for the condition by group interaction $\left(F[1,68]=16.285, P<0.001, \eta^{2}=0.193\right)$. A multivariate analysis with the group as a fixed factor and the second physical ToM condition as a covariate was performed to examine the relationship between the second Cog ToM condition and second Aff ToM condition. The EOS group had significantly lower scores in the second order Cog ToM $\left(F[1,68]=16.737, P<0.001, \eta^{2}=0.200\right)$ and second order Aff $\operatorname{ToM}\left(F[1,68]=13.631, P<0.001, \eta^{2}=0.169\right)$ (Figure 1).

\section{FPR task}

Almost all participants answered the control questions of the Faux Pas Recognition Test correctly $(F[1,68]=3.058$, $\left.P=0.085, \eta^{2}=0.043\right)$. The MANOVA found significant group differences in recognition of faux pas $(F[1,68]=4.395$, $\left.P=0.041, \eta^{2}=0.060\right)$ and understanding of faux pas $(F[1,68]$ $=7.929, P=0.006, \eta^{2}=0.104$ ) (Figure 2, Table 2).

\section{Correlation coefficients among multi- factors or patient groups}

Table 3 presents the correlation of the illness course, symptoms, executive function, and chlorpromazine equivalents with the scores of the Cog and Aff ToMs. There was no significant association between the symptom clusters, illness duration, chlorpromazine equivalents, or ToM scores. There was a significant association between SCWT-C performance and second-order-ToM score (Table 3).

\section{Discussion}

This study investigated the ToM performance in AOS using the Yoni task and FRP test compared with HC group, and examined whether the ToM scores were related to the course of the disease and the PANSS scores. Results indicated that patients with AOS demonstrated impairment to different 

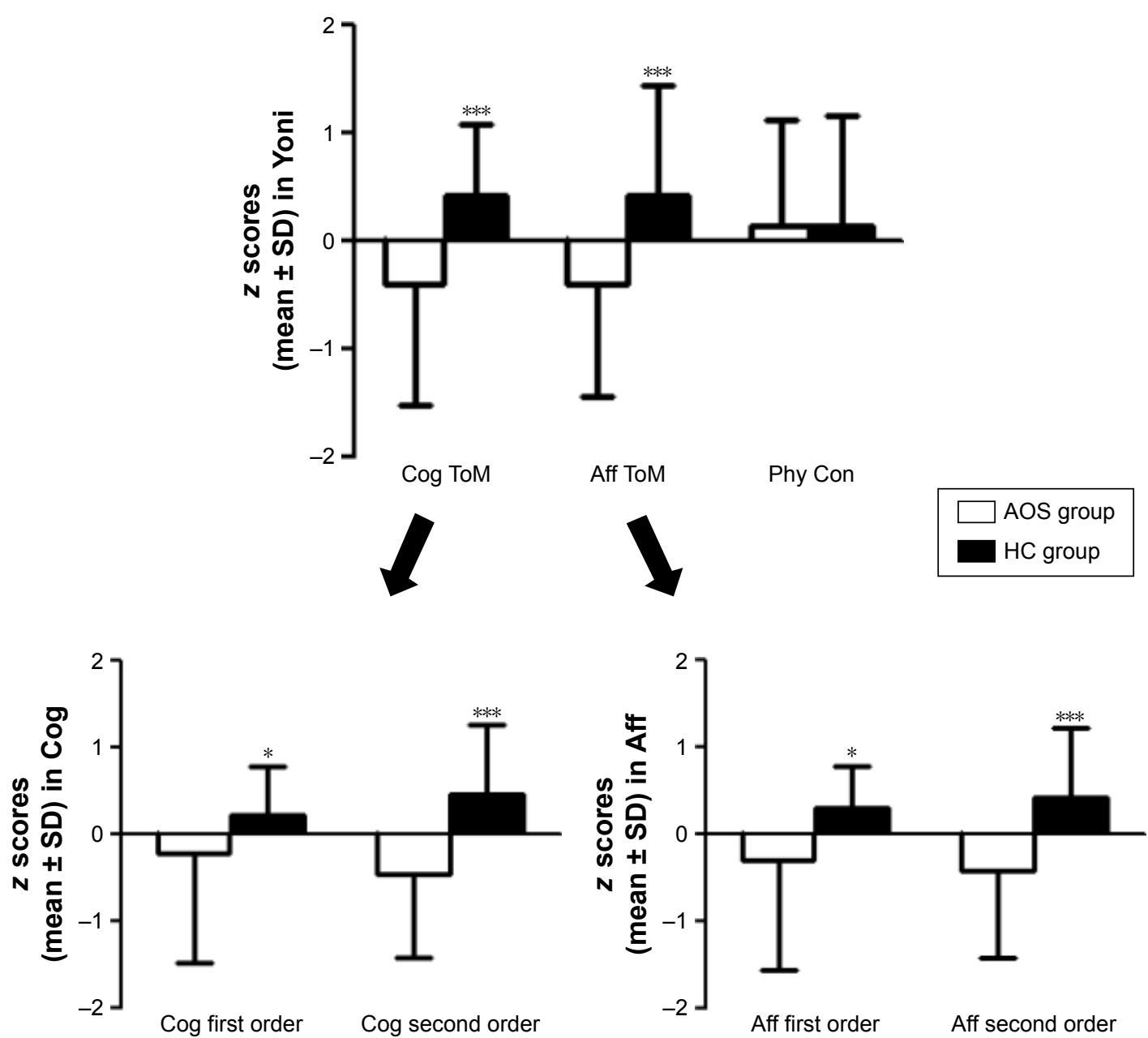

Figure I The three parts of $z$ scores in Yoni task.

Notes: Mean score of Cog ToM, Aff ToM and Phy Con for subjects with AOS and HC in Yoni task. The error bars: SD; $* P<0.05$ for differences between the two groups. $* * * P<0.001$.

Abbreviations: Aff, affective; AOS, adolescent onset schizophrenia; Cog, cognitive; HCs, healthy controls; Phy Con, physical condition; ToM, Theory of Mind.

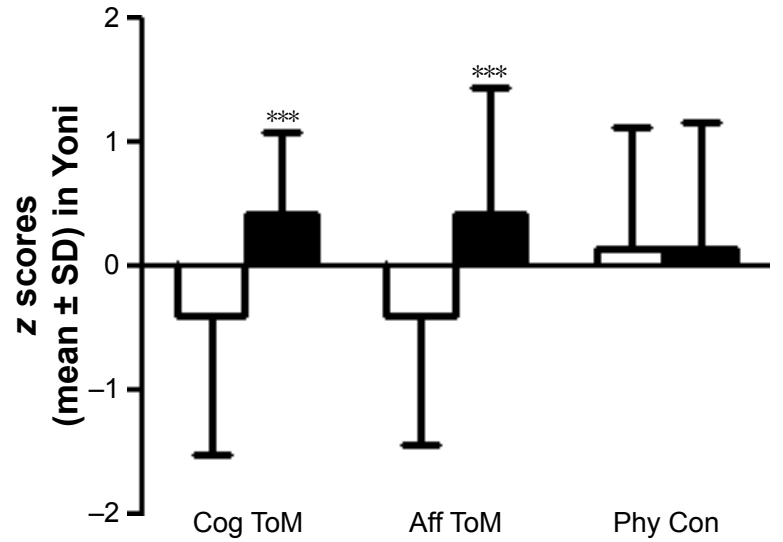

AOS group

HC group

Figure 2 The sub-tests of $z$ scores in FPR.

Notes: Mean score of Cog ToM, Aff ToM and Phy Con for subjects with AOS and $\mathrm{HC}$ in FPR. The error bars: $\mathrm{SD} ; * * * P<0.001$.

Abbreviations: Aff, affective; AOS, adolescent onset schizophrenia; Cog, cognitive; FPR, Faux Pas Recognition; HCs, healthy controls; Phy Con, physical condition; SD, standard deviation; ToM, Theory of Mind. extents of the Cog and Aff ToM compared with age-, gender, and intelligence-matched healthy controls. In the Yoni task, patients with AOS showed differences in ToM abilities either on a different order or under different conditions. It seemed that impairment in second-order-ToM is more serious. The FPR task results revealed that AOS patients were not always able to recognize a faux pas or understand complicated emotions under the faux pas scenario. Furthermore, as indicated by the correlation analysis, neither Cog ToM nor Aff ToM was related to the patients' symptoms, disease duration, dose of medication, and IQ.

With regard to previous ToM studies in schizophrenia patients, our results are in concordance with the meta-analysis by Bora et al that found that individuals with schizophrenia seemed to perform poorly on both Cog and Aff ToM tasks. ${ }^{19}$ However, Shamay-Tsoory et al used the same Yoni task and found the Aff ToM component was damaged but that the Cog ToM was intact in AS patients. ${ }^{29}$ Thus, Shamay-Tsoory 
Table 2 Performances of AOS, control group in the Yoni task and Faux Pas Recognition Test

\begin{tabular}{|c|c|c|c|c|c|c|c|}
\hline \multirow[t]{2}{*}{ Variables } & \multicolumn{2}{|c|}{$\begin{array}{l}\text { AOS } \\
(n=35)\end{array}$} & \multicolumn{2}{|c|}{$\begin{array}{l}\mathrm{HC} \\
(n=35)\end{array}$} & \multirow[t]{2}{*}{$\boldsymbol{F}$} & \multirow[t]{2}{*}{$P$-value } & \multirow[t]{2}{*}{$\eta^{2}$} \\
\hline & Mean & SD & Mean & SD & & & \\
\hline \multicolumn{8}{|l|}{ Yoni task } \\
\hline \multicolumn{8}{|l|}{ First-order-ToM } \\
\hline First order cognitive ToM & -0.22 & 1.26 & 0.22 & 0.56 & 4.031 & $0.049 *$ & 0.057 \\
\hline First order affective ToM & -0.30 & 1.27 & 0.30 & 0.47 & 6.809 & $0.011^{*}$ & 0.092 \\
\hline \multicolumn{8}{|l|}{ Second-order-ToM } \\
\hline Second order cognitive ToM & -0.46 & 0.99 & 0.46 & 0.78 & 16.737 & $<0.001 * * *$ & 0.200 \\
\hline Second order affective ToM & -0.42 & 1.00 & 0.42 & 0.80 & $|3.63|$ & $<0.00 I^{* * *}$ & 0.169 \\
\hline \multicolumn{8}{|l|}{ Faux Pas Recognition Test } \\
\hline Faux Pas Recognition questions (cognitive ToM) & -0.17 & 1.04 & 0.17 & 0.94 & 4.395 & $0.04 I^{*}$ & 0.060 \\
\hline Faux Pas understanding (affective ToM) & -0.36 & 0.76 & 0.39 & 1.09 & 7.929 & $0.006 * *$ & 0.104 \\
\hline
\end{tabular}

Notes: $P<0.05$ for differences between the two groups. $* P<0.05 ; * * P<0.01 ; * * P<0.001$.

Abbreviations: AOS, adolescent onset schizophrenia; HC, healthy controls; SD, standard deviation; ToM, Theory of Mind.

et al proposed that Aff ToM impairment instead of complete ToM impairment in schizophrenia was a core characteristic. Based on the results in our study, it may not be plausible to conclude that Aff ToM impairment is a core characteristic of schizophrenia, at least not for late AOS.

The present study found a lower significance level in first order Cog ToM impairment $(P=0.049)$ but a higher significance level in second order Cog ToM impairment between AOS and controls, demonstrating that second order Cog ToM showed more severe damage in AOS patients. Some previous studies found AS patients with relatively intact Cog ToM, but the current results showed AOS with higher order Cog ToM damaged, implying that earlier onset schizophrenia was associated with more severe ToM damage. This finding may be a major reason why AOS patients have poor outcomes. ${ }^{30}$

The meta-analysis data supported the hypothesis that ToM was subserved by a network of regions that included the MPFC, temporo-parietal junction, precuneus, and superior temporal sulcus. ${ }^{31}$ These regions played distinct roles during ToM. The vmPFC is considered to be closely related to Aff ToM, whereas the dlPFC is believed to be more related to Cog ToM. ${ }^{32,33}$ Importantly, an elaborated ToM continues beyond the beginning of puberty until early adulthood. ${ }^{34}$ For instance, the MPFC develops during late childhood and early adolescence. More developmental changes in neural activities were observed in the vmPFC in adolescents. ${ }^{35}$ The non-linear changes in vmPFC activities that occur between 10 and 12 and 14 and 16 years of age suggest that the vmPFC develops unsteadily in adolescents around the onset of puberty. ${ }^{36}$ The adolescent brain is still undergoing several maturational processes, which makes it more vulnerable to some of the effects of the disease. Therefore, schizophrenia onset during this period may lead to a disruption in neurodevelopment, which may lead to a worse effect in adolescents.

Pearson correlations of the ToM (FPR and Yoni tasks) subscores with PANSS and the course of disease, the StroopColor Word Test (SWCT)-C performance, IQ, and dosage of drugs in AOS group are reported in Table 3. ToM deficits

Table 3 Correlation coefficients between symptom, illness duration, EF, CPZ equivalents and results of ToM for AOS

\begin{tabular}{|c|c|c|c|c|c|c|}
\hline \multirow[t]{2}{*}{ Variables } & \multicolumn{2}{|c|}{ Faux Pas Recognition Test } & \multicolumn{4}{|c|}{ Yoni task } \\
\hline & Cog & Aff & CogI & $\operatorname{Cog} 2$ & Affl & Aff2 \\
\hline IQ-Raven (correct number) & -0.16 & -0.18 & 0.17 & -0.16 & -0.04 & -0.25 \\
\hline SCWT-C (EF) & 0.13 & 0.24 & -0.25 & $-0.44 * *$ & -0.31 & $-0.59 * *$ \\
\hline CPZ equivalents (mg) & 0.07 & -0.11 & -0.02 & 0.12 & -0.20 & 0.15 \\
\hline Disease duration & -0.11 & -0.02 & 0.07 & -0.13 & -0.02 & -0.13 \\
\hline \multicolumn{7}{|l|}{ PANSS subscales } \\
\hline Positive symptoms & -0.07 & 0.04 & 0.12 & 0.05 & -0.10 & -0.04 \\
\hline Negative symptoms & -0.06 & -0.02 & -0.25 & -0.17 & -0.25 & -0.01 \\
\hline General symptoms & -0.02 & 0.02 & -0.16 & -0.24 & -0.28 & -0.17 \\
\hline Total & 0.08 & 0.09 & -0.19 & -0.17 & -0.25 & -0.14 \\
\hline
\end{tabular}

Notes: EF: **P<0.0I; Cog: Faux Pas Recognition questions; Aff: Faux Pas understanding; Cogl: first order cognitive ToM; Aff: first order affective ToM; Cog2: second order cognitive ToM; Aff2: second order affective ToM.

Abbreviations: Aff, affective; AOS, adolescent onset schizophrenia; Cog, cognitive; CPZ, chlorpromazine; EF, executive function; IQ, intelligent quotient; PANSS, Positive and Negative Syndrome Scale; SCWT, Stroop-Color Word Test; ToM, Theory of Mind. 
are independent of the course and severity of disease, and the results were in agreement with the study by Bora et al, which showed mental impairment in patients with first-episode schizophrenia and their unaffected siblings. ${ }^{37}$ Another study demonstrated that deficits in social cognition existed in the prodromal stage of schizophrenia and that ToM deficits in schizophrenia might precede the initial stage of psychotic breakdown, thereby accelerating disease progression. ${ }^{38}$ Therefore, the present results seemed to be more plausible to infer that ToM damage in schizophrenia may be a trait phenomenon, but not a state one, together with insignificance of the relativity between ToM performance and dosage of drugs, our results added the evidence that ToM damage may be an intermediate phenotype of schizophrenia. ${ }^{38}$ Although the AOS and HC groups were matched for age, gender, IQ, and education level, the AOS group had significantly poorer executive performance (eg, SWCT). These behavioral findings are corroborated by emerging evidence that high-order Cog ToM involves executive functions. ${ }^{39}$ Our results on the significant relativity between SCWT-C performance and second-order-ToM were indeed in accordance with the above findings, whereas ToM is not simply a more general executive function. ${ }^{39}$ Indeed, ToM performance requires more processes than executive functions.

\section{Limitations}

The limitations of the study should be considered. Our subjects were young and newly exposed to this chronic disease, and AOS had a shorter disease course (approximately 2-3 years). The relationship between disease progression and the severity of the ToM injury is uncertain. More AOS patients with longer courses should be recruited in future studies. Although our study strictly controlled for the level of intelligence, gender, education, background and type and dose of drugs, we should not ignore the influence of medications. Medications may have variable effects on shaping brain activities and performance, and various doses may also affect basic social cognitive functions. Such small sample sizes of the AOS may not be representative of AOS as a whole. In addition, the current work does not directly compare the ability of ToM in AOS with AS so as to identify the special property in AOS. Adolescence can be divided into three stages (the early, middle, and late stages), with the brain maturation processes continuing throughout the three stages. In our study, we chose only subjects in late adolescence. We will continue our study of the disease impacts on the ToM in the two other stages of adolescence.

\section{Acknowledgments}

This study is sponsored by the National Natural Science Foundation of China (nos 31571149, 91432301, 81300944, 81301176, and 91232717) and Natural Science Foundation of Anhui Province (1308085QH148). We are deeply grateful to all the patients and healthy controls who participated in this research.

\section{Disclosure}

The authors report no conflicts of interest in this work.

\section{References}

1. Eisenberg L. The course of childhood schizophrenia. AMA Arch Neurol Psychiatry. 1957;78(1):69-83.

2. Armenteros JL, Davies M. Antipsychotics in early onset schizophrenia: systematic review and meta-analysis. Eur Child Adolesc Psychiatry. 2006;15(3):141-148.

3. Young CM, Findling RL. Pharmacologic treatment of adolescent and child schizophrenia. Expert Rev Neurother. 2004;4(1):53-60.

4. Schulz SC, Findling RL, Wise A, Friedman L, Kenny J. Child and adolescent schizophrenia. Psychiatr Clin North Am. 1998;21(1):43-56.

5. Hans SL, Auerbach JG, Asarnow JR, Styr B, Marcus J. Social adjustment of adolescents at risk for schizophrenia: the Jerusalem Infant Development Study. J Am Acad Child Adolesc Psychiatry. 2000;39(11): 1406-1414.

6. Basso MR, Nasrallah HA, Olson SC, Bornstein RA. Cognitive deficits distinguish patients with adolescent- and adult-onset schizophrenia. Neuropsychiatry Neuropsychol Behav Neurol. 1997;10(2):107-112.

7. Rohde G, Klein W, Arinir U, et al. Association of the ASP299GLY TLR4 polymorphism with COPD. Respir Med. 2006;100(5):892-896.

8. Ping-Chia L, I-Ju L, Yu-Ching L, Li-Ching C, Wen-Chung C. Substance $P$ scavenger enhances antioxidant defenses and prevents prothrombotic effects on the rat lung after acute exposure to oil smoke. J Biomed Sci. 2009; $16: 58$.

9. Fett AK, Viechtbauer W, Dominguez MD, Penn DL, van Os J, Krabbendam L. The relationship between neurocognition and social cognition with functional outcomes in schizophrenia: a meta-analysis. Neurosci Biobehav Rev. 2011;35(3):573-588.

10. Brothers L, Ring B. A neuroethological framework for the representation of minds. J Cogn Neurosci. 1992;4(2):107-118.

11. Shamay-Tsoory SG, Harari H, Aharon-Peretz J, Levkovitz Y. The role of the orbitofrontal cortex in affective theory of mind deficits in criminal offenders with psychopathic tendencies. Cortex. 2010;46(5): 668-677.

12. Ho KK, Lui SS, Hung KS, et al. Theory of mind impairments in patients with first-episode schizophrenia and their unaffected siblings. Schizophr Res. 2015;166(1-3):1-8.

13. Perner J, Wimmer H. "John thinks that Mary thinks that ..." attribution of second-order beliefs by 5- to 10-year-old children. J Exp Child Psychol. 1985;39(3):437-471.

14. Baron-Cohen S, O'Riordan M, Stone V, Jones R, Plaisted K. Recognition of faux pas by normally developing children and children with Asperger syndrome or high-functioning autism. J Autism Dev Disord. 1999;29(5):407-418.

15. Vogeley K, Bussfeld P, Newen A, et al. Mind reading: neural mechanisms of theory of mind and self-perspective. Neuroimage. 2001; 14(1 Pt 1):170-181.

16. Steinberg L. Cognitive and affective development in adolescence. Trends Cogn Sci. 2005;9(2):69-74.

17. Ruffman T, Keenan TR. The belief-based emotion of surprise: the case for a lag in understanding relative to false belief. Dev Psychol. 1996;32(1):40-49. 
18. Rieffe C, Terwogt MM, Cowan R. Children's understanding of mental states as causes of emotions. Infant Child Dev. 2005; 14(3):259-272.

19. Bora E, Yucel M, Pantelis C. Theory of mind impairment: a distinct trait-marker for schizophrenia spectrum disorders and bipolar disorder? Acta Psychiatr Scand. 2009;120(4):253-264.

20. Corcoran R, Mercer G, Frith CD. Schizophrenia, symptomatology and social inference: investigating "theory of mind" in people with schizophrenia. Schizophr Res. 1995;17(1):5-13.

21. Green MF, Bearden CE, Cannon TD, et al. Social cognition in schizophrenia, Part 1: performance across phase of illness. Schizophr Bull. 2012;38(4):854-864.

22. Gil D, Fernandez-Modamio M, Bengochea R, Arrieta M. Adaptation of the Hinting Task theory of the mind test to Spanish. Rev Psiquiatr Salud Ment. 2012;5(2):79-88.

23. Hamilton M. A rating scale for depression. J Neurol Neurosurg Psychiatry. 1960;23:56-62.

24. Hamilton M. The assessment of anxiety states by rating. $\mathrm{Br} J \mathrm{Med}$ Psychol. 1959;32(1):50-55.

25. Kay SR, Fiszbein A, Opler LA. The Positive and Negative Syndrome Scale (PANSS) for schizophrenia. Schizophr Bull. 1987;13(2):261-276.

26. Gardner DM, Murphy AL, O'Donnell H, Centorrino F, Baldessarini RJ. International consensus study of antipsychotic dosing. Am J Psychiatry. 2010;167(6):686-693.

27. Troyer AK, Moscovitch M, Winocur G. Clustering and switching as two components of verbal fluency: evidence from younger and older healthy adults. Neuropsychology. 1997;11(1):138-146.

28. Zhu CY, Lee TM, Li XS, Jing SC, Wang YG, Wang K. Impairments of social cues recognition and social functioning in Chinese people with schizophrenia. Psychiatry Clin Neurosci. 2007;61(2):149-158.

29. Shamay-Tsoory SG, Aharon-Peretz J, Perry D. Two systems for empathy: a double dissociation between emotional and cognitive empathy in inferior frontal gyrus versus ventromedial prefrontal lesions. Brain. 2009;132(Pt 3):617-627.
30. Brune M. "Theory of mind" in schizophrenia: a review of the literature. Schizophr Bull. 2005;31(1):21-42.

31. Dodell-Feder D, Koster-Hale J, Bedny M, Saxe R. fMRI item analysis in a theory of mind task. Neuroimage. 2011;55(2):705-712.

32. Shamay-Tsoory SG, Tibi-Elhanany Y, Aharon-Peretz J. The ventromedial prefrontal cortex is involved in understanding affective but not cognitive theory of mind stories. Soc Neurosci. 2006;1(3-4):149-166.

33. Burnett S, Sebastian C, Cohen Kadosh K, Blakemore SJ. The social brain in adolescence: evidence from functional magnetic resonance imaging and behavioural studies. Neurosci Biobehav Rev. 2011; 35(8):1654-1664.

34. Vetter NC, Leipold K, Kliegel M, Phillips LH, Altgassen M. Ongoing development of social cognition in adolescence. Child Neuropsychol. 2013;19(6):615-629.

35. Vetter NC, Weigelt S, Dohnel K, Smolka MN, Kliegel M. Ongoing neural development of affective theory of mind in adolescence. Soc Cogn Affect Neurosci. 2014;9(7):1022-1029.

36. Moor BG, Macks ZA, Guroglu B, Rombouts SA, Molen MW, Crone EA. Neurodevelopmental changes of reading the mind in the eyes. Soc Cogn Affect Neurosci. 2012;7(1):44-52.

37. Bora E, Yucel M, Pantelis C. Theory of mind impairment in schizophrenia: meta-analysis. Schizophr Res. 2009;109(1-3):1-9.

38. Chung YS, Kang DH, Shin NY, Yoo SY, Kwon JS. Deficit of theory of mind in individuals at ultra-high-risk for schizophrenia. Schizophr Res. 2008;99(1-3):111-118.

39. Fernandez-Gonzalo S, Pousa E, Jodar M, Turon M, Duno R, Palao D. Influence of the neuropsychological functions in theory of mind in schizophrenia: the false belief/deception paradigm. J Nerv Ment Dis. 2013;201(7):609-613.
Neuropsychiatric Disease and Treatment

\section{Publish your work in this journal}

Neuropsychiatric Disease and Treatment is an international, peerreviewed journal of clinical therapeutics and pharmacology focusing on concise rapid reporting of clinical or pre-clinical studies on a range of neuropsychiatric and neurological disorders. This journal is indexed on PubMed Central, the 'PsycINFO' database and CAS,

\section{Dovepress}

and is the official journal of The International Neuropsychiatric Association (INA). The manuscript management system is completely online and includes a very quick and fair peer-review system, which is all easy to use. Visit http://www.dovepress.com/testimonials.php to read real quotes from published authors. 